A. IKEDA

KODAI MATH. J.

18 (1995), $57-67$

\title{
ON THE SPECTRUM OF HOMOGENEOUS SPHERICAL SPACE FORMS
}

\author{
Dedicated to Professor Hideki Ozeki on the occasion of \\ his sixtieth birthday
}

AKIRA IKEDA

\section{Introduction}

Let $S^{2 n-1}(n>1)$ be the $(2 n-1)$-dimensional sphere of constant curvature 1 and $G$ be a finite subgroup of the orthogonal group of degree $2 n$ acting fixed point freely on $S^{2 n-1}$. Then the spherical space form $M=S^{2 n-1} / G$ has a constant curvature 1 . If $G$ is cyclic then the spherical space form is called a lens space. The Laplacian $\Delta$ acting on the space of smooth functions on a spherical space form has a discrete spectrum with finite multiplicities. It is well known that the eigenvalues of the Laplacian $\Delta$ are of the form $k(k+2 n-2)(k=0,1,2, \cdots)$. Let $a_{k}$ be the multiplicity of eigenvalue $k(k+2 n-2)$. The Poincare series $\sum_{k=0}^{\infty} a_{k} z^{k}$ associated to the spectrum for homogeneous spherical space becomes a rational function which is a nice form to study the spectrum of spherical space forms and has been studied by the author in order to construct riemannian manifolds which are isospectral but not isometric. On the other hand, the classification for homogeneous spherical space forms is given by Wolf [4]. Wolf's classification theorem for homogeneous space forms states that for any $g \in G$ of a homogeneous spherical space form $S^{2 n-1} / G$, there is a unimodular complex number $\lambda$ such that half the eigenvalues of $g$ are $\lambda$ and the other half are $\bar{\lambda}$. By this theorem, the Poincaré series associated to the spectrum for homogeneous spherical space forms becomes a simple form.

In this paper using this form we give the spectrum of homogeneous spherical space forms explicitly. For the cases of the spheres and homogeneous lens spaces their spectra are given in M. Berger [1] and in T. Sakai [3]. Our table for the spectrum of homogeneous lens spaces is different from Sakai [3], and is more simpler than Sakai's table.

\section{Preliminary}

In this section we give elementary formulae which we need in the following sections. The following two lemmas are well known.

Received January 6, 1994. 
LEMMA 2.1. We have $(1-z)^{-n}=\sum_{k=0}^{\infty}\left(\begin{array}{c}n+k-1 \\ k\end{array}\right) z^{k}$.

LEMMA 2.2. Let $\lambda$ be a $q$-th root of one. Then

$$
\sum_{i=1}^{q} \lambda^{2}= \begin{cases}0 & \text { if } \lambda \neq 1, \\ q & \text { if } \lambda=1 .\end{cases}
$$

Using the above two Lemmas, we get

LEMMA 2.3. Let $\lambda$ be a unimodular complex number, $|\lambda|=1$. The

$$
(1-\lambda z)^{-n}(1-\bar{\lambda} z)^{-n}=\sum_{t=0}^{\infty} \sum_{l=0}^{t}\left(\begin{array}{c}
n+t-l-1 \\
n-1
\end{array}\right)\left(\begin{array}{c}
n+l-1 \\
n-1
\end{array}\right) \lambda^{t-2 l} z^{t} .
$$

Let $\lambda$ be a primitive $q$-th root of one. Define

$$
F_{q}(n, z)=\frac{1}{q} \sum_{i=1}^{q}\left(1-z^{2}\right)\left(1-\lambda^{2} z\right)^{-n}\left(1-\bar{\lambda}^{2} z\right)^{-n} .
$$

Then we obtain easily by using Lemma 2.2 and Lemma 2.3

Proposition 2.4. Let $\lambda$ and $F_{q}(n, z)$ be as above. Then we have

$$
F_{q}(n, z)=1+\sum_{k=2}^{\infty} \sum_{k \equiv 2 l(q), 0 \leqq l \leqq k} \frac{n-1+k}{n-1}\left(\begin{array}{c}
n-2+k-l \\
n-2
\end{array}\right)\left(\begin{array}{c}
n-2+l \\
n-2
\end{array}\right) z^{k} .
$$

Define $a_{k}(n, q)$ by

$$
F_{q}(n, z)=\sum_{k=0}^{\infty} a_{k}(n, q) z^{k}
$$

Then

$$
\left\{\begin{array}{l}
a_{0}(n, q)=1, \\
a_{1}(n, q)=0, \\
a_{k}(n, q)=\frac{n-1+k}{n-1} \sum_{k \equiv 2 l(q), 0 \leqq l \leqq k}\left(\begin{array}{c}
n-2+k-l \\
n-2
\end{array}\right)\left(\begin{array}{c}
n-2+l \\
n-1
\end{array}\right) \quad k \geqq 2 .
\end{array}\right.
$$

Proposition 2.5. Let $a_{k}(n, q)$ be as above. Then

(1) If $q$ is odd, then $a_{k}(n, q) \neq 0$ if and only if $k$ is even or $k$ is odd with $\geqq q$.

(2) If $q$ is even, then $a_{k}(n, q) \neq 0$ if and only if $k$ is even.

Proof. By Proposition 2.4, $a_{k}(n, z) \neq 0$ if and only if the congruence equation $k \equiv 2 l(q)(0 \leqq l \leqq k)$ has a solution. Hence our assertions are easy to see.

q.e.d.

We use the following Lemma 2.6 in 4 . 
LEMMA 2.6. For $n>1,\left(\begin{array}{c}2 n-2+k+t \\ 2 n-2\end{array}\right)\left(\begin{array}{c}2 n-2+k-t \\ 2 n-2\end{array}\right)$ is strictly decreasing with respect to $t(0 \leqq t \leqq k)$.

Proof. An easy computation. q.e.d.

\section{Homogeneous spherical space forms}

The classification of homogeneous spherical space forms has been obtained by J.A. Wolf [4]. In this section we describe his results and study some properties of the finite subgroups appearing in the classification. Let $S O(3)$ be the special orthogonal group acting on the Euclidean 3 -space $R^{3}$. It is well known that the finite subgroups of $S O(3)$ (up to conjugations in $S O(3)$ ) are given as follows (for details, see [4]);

$$
\begin{gathered}
\boldsymbol{Z}_{m}: \text { the cyclic group of order } m(m \geqq 1), \\
\boldsymbol{D}_{2 m}: \text { the dihedral group of order } 2 m(m \geqq 2), \\
\boldsymbol{T}: \text { the tetrahedral group of order } 12, \\
\boldsymbol{O}: \text { the octahedral group of order } 24, \\
\boldsymbol{I}: \text { the icosahedral group of order } 60 .
\end{gathered}
$$

Let $S p(1)$ be the group of unit quaternions and $\pi: S p(1) \rightarrow S O(3)$ the universal covering map. The binary dihedral and binary polyhedral groups are defined by

$$
\boldsymbol{D}_{\dot{4} m}^{*}=\pi^{-1}\left(\boldsymbol{D}_{2 m}\right), \quad \boldsymbol{T}^{*}=\pi^{-1}(\boldsymbol{T}), \quad \boldsymbol{O}^{*}=\pi^{-1}(\boldsymbol{O}), \quad \boldsymbol{I}^{*}=\pi^{-1}(\boldsymbol{I}) .
$$

$\boldsymbol{T}^{*}, \boldsymbol{O}^{*}$ and $\boldsymbol{I}^{*}$ are called the binary tetrahedral, binary octahedral, binary icosahedral groups, respectively.

We divide the binary dihedral and binary polyhedral groups into cyclic subgroups as follows;

Proposition 3.1. (1) $\boldsymbol{D}_{4 m}^{*}$ is the sum of the sets consisting of one cyclic group of order $2 m$ and $m$ cyclic subgroups of order 4 . Any two subgroups have a common cyclic subgroup of order 2 which is the center of $S p(1)$. (2) $T^{*}$ is the sum of the sets consisting of 4 cyclic subgroups of order 6 and 3 cyclic subgroups of order 4. Any two subgroups have a common cyclic subgroup of order 2 which is the center of $S p(1)$. (3) $O *$ is the sum of the sets consisting of 3 cyclic subgroups of order 8,4 cyclic subgroups of order 6 and 6 cyclic subgroups of order 4. Any two subgroups have a common cyclic subgroup of order 2 which is the center of $S p(1)$. (4) $\boldsymbol{I}^{*}$ is the sum of the sets consisting of 6 cyclic subgroups of order 10, 10 cyclic subgroups of order 6 and 15 cyclic subgroups of order 4 . Any two subgroups have a common cyclic subgroup of order 2 which is the center of $S p(1)$. 
Proof. We shall show (2). The tetrahedral group $\boldsymbol{T}$ consists of symmetries of regular tetrahedron. There are two types of symmetries. One is a rotation about the line through a vertex of the tetrahedron and the center of the face opposite the vertex. Other one is a rotation by $\pi$ about the line through midpoints of two edges which are in opposite side to each other. From these facts, (2) is easy to see. By the same way as above, we can show (1), (3) and (4).

q.e.d.

The following classification theorem can be found in Wolf [4].

THEOREM 3.2. Let $G$ be a fixed point free finite subgroup of $S O(2 n)(n>1)$. Then the following conditions are equivalent.

(1) $S^{2 n-1} / G$ is a Riemannian homogeneous space.

(2) For any $g \in G$, either $g= \pm 1_{2 n}$ or there is a unimodular complex number $\lambda$ such that half the eigenvalues of $g$ are $\lambda$ and the other half are $\bar{\lambda}$.

(3) Either (i) $G$ is cyclic of order $q \geqq 1$, and $G$ is conjugate to the image of $\boldsymbol{Z}_{q}=\left\{g^{t}\right\}_{t=0}^{q-1}$ under the representation $\boldsymbol{\tau} \oplus \cdots \oplus \boldsymbol{\tau}(n$-times $)$ of $\boldsymbol{Z}_{q}$, where $\tau\left(g^{t}\right)=$ $\left(\begin{array}{rr}\cos (2 \pi t / q) & \sin (2 \pi t / q) \\ -\sin (2 \pi t / q) & \cos (2 \pi t / q)\end{array}\right) \subset S O(2)$, or (ii) $G$ is isomorphic to a binary dihedral or binary polyhedral group $P^{*}, n$ is even and $G$ is conjugate to the image of $P^{*}$ under the representation $\rho \oplus \cdots \oplus \rho(n / 2$-times $)$ of $P^{*}$, where $\rho: P^{*} \subset S p(1) \subset$ $S O(4)$.

From the above Theorem, we can list homogeneous spherical space forms as follows;

(1) $\quad M=S^{2 n-1} / \boldsymbol{Z}_{m} \quad(m \geqq 1)$, where $\boldsymbol{Z}_{m}$ is generated by $e^{2 \pi \imath / m}$ and acts on $R^{2 n}=C^{n}$ by multiplication on each complex coordinate ;

(2) $\quad M=S^{4 n-1} / \boldsymbol{D}_{4 m}^{*}(m \geqq 2)$, where $\boldsymbol{D}_{4 m}^{*}$ acts on $R^{4 n}=H^{n}$ by multiplication on each quaternionic coordinate from the left;

(3) $M=S^{4 n-1} / \boldsymbol{T}^{*}$, where $\boldsymbol{T}^{*}$ acts on $R^{4 n}=H^{n}$ by multiplication on each quaternionic coordinate from the left;

(4) $\quad M=S^{4 n-1} / O^{*}$, where $O^{*}$ acts on $R^{4 n}=H^{n}$ by multiplication on each quaternionic coordinate from the left;

(5) $\quad M=S^{4 n-1} / I^{*}$, where $I^{*}$ acts on $R^{4 n}=H^{n}$ by multiplication on each quaternionic coordinate from the left.

\section{Spectra of homogeneous spherical space forms}

In this section we start with recalling the Poincaré Series associated to the spectrum of a spherical space form. For details of this section, refer to [2].

Let $M=S^{2 n-1} / G$ be a $(2 n-1)$-dimensional spherical space form and $\Delta$ the Laplacian acting on the smooth functions on $M$. Then each eigenvalue of $\Delta$ is of the form $k(k+2 n-2)(k=0,1,2, \cdots)$. Let $a_{k}$ be the multiplicity of eigenvalue $k(k+2 n-2)$. We defined the Poincaré series associated to the spectrum of $\Delta$ by 


$$
F_{G}(z)=\sum_{k=0}^{\infty} a_{k} z^{k}
$$

Then this function has the representative of a rational function [2].

Proposition 4.1. We have

$$
F_{G}(z)=\frac{1}{|G|} \sum_{g \in G} \frac{1-z^{2}}{\operatorname{det}\left(z 1_{2 n}-g\right)} .
$$

where $|G|$ denotes the order of $G$.

Let $S^{2 n-1} / G$ be a homogeneous spherical space form. Let $\boldsymbol{Z}_{q}$ be a cyclic subgroup of order $q$ in $G$ and $\lambda$ a primitive $q$-th root of one. By (2) in Theorem 3.1 , we have

$$
\begin{aligned}
\sum_{g \in Z_{q}} \frac{1-z^{2}}{\operatorname{det}\left(z 1_{2 n}-g\right)} & =\left(1-z^{2}\right) \sum_{k=1}^{q}\left(1-\lambda^{k} z\right)^{-n}\left(1-\bar{\lambda}^{k} z\right)^{-n} \\
& =q F_{q}(n, z) .
\end{aligned}
$$

(I) $S^{2 n-1} / Z_{q}(q \geqq 1)$; homogeneous lens spaces.

The Poincaré series is

$$
F_{Z_{q}}(z)=F_{q}(n, z)=\frac{1}{q} \sum_{i=1}^{q}\left(1-z^{2}\right)\left(1-\lambda^{2} z\right)^{-n}\left(1-\bar{\lambda}^{i} z\right)^{-n},
$$

where $\lambda$ is a primitive $q$-th root of one.

By Proposition 2.4, the coefficients $a_{k}$ 's of the Poincaré series $F_{Z_{q}}(z)$ are

$$
\left\{\begin{array}{l}
a_{0}=1, \\
a_{1}=0, \\
a_{k}=\frac{n-1+k}{n-1} \sum_{k \equiv 2 l(q), 0 \leqq l \leqq k}\left(\begin{array}{c}
n-2+k-l \\
n-2
\end{array}\right)\left(\begin{array}{c}
n-2+l \\
n-1
\end{array}\right) \quad k \geqq 2 .
\end{array}\right.
$$

By Proposition 2.4 and 2.5, we have

THEOREM 4.2 (cf. [3]). Let $S^{2 n-1} / Z_{q}(q \geqq 2)$ be the homogeneous lens space. Then eigenvalues of Laplacian for the lens space are

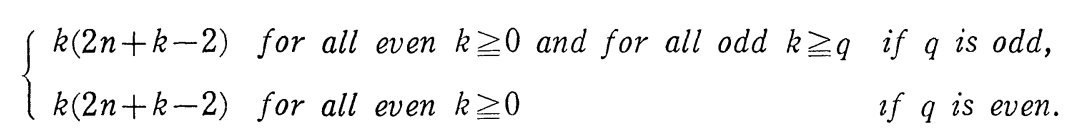

If $k(2 n+k-2)$ is an eigenvalue, then its multiplicity $a_{k}$ is

$$
a_{k}=\frac{n-1+k}{n-1} \sum_{k \equiv 2 l(q), 0 \leq l \leqq k}\left(\begin{array}{c}
n-2+k-l \\
n-2
\end{array}\right)\left(\begin{array}{c}
n-2+l \\
n-2
\end{array}\right) .
$$

(II) $S^{4 n-1} / G$ where $G$ is a binary dihedral group $\boldsymbol{D}_{4 m}^{*}$ of order $4 m(m \geqq 2)$.

By (1) in Proposition 3.1, the group $D_{4 m}^{*}$ consists of the cyclic subgroup 
$\boldsymbol{Z}_{2 m}$ of order $2 m$ and $m$ cyclic subgroups $\boldsymbol{Z}_{4}$ of order 4 . The intersection group of any two of these cyclic subgroups is a cyclic group of order 2 which is the kernel of $\pi: \boldsymbol{D}_{4 m}^{*} \rightarrow \boldsymbol{D}_{2 m}$. Thus we have

$$
\begin{aligned}
F_{G}(z) & =\frac{1}{4 m}\left\{2 m F_{2 m}(2 n, z)+4 m F_{4}(2 n, z)-2 m F_{2}(2 n, z)\right\} \\
& =\frac{1}{2} F_{2 m}(2 n, z)+F_{4}(2 n, z)-\frac{1}{2} F_{2}(2 n, z) .
\end{aligned}
$$

By Proposition 2.5, $a_{2 k-1}=0(k \geqq 1)$. We compute $a_{2 k}$

$$
\begin{aligned}
& a_{2 k}= \frac{2 n-1+2 k}{2 n-1}\left\{\frac{1}{2} \sum_{l \equiv k(m), 0 \leqq l \leqq 2 k}\left(\begin{array}{c}
2 n-2+2 k-l \\
2 n-2
\end{array}\right)\left(\begin{array}{c}
2 n-2+l \\
2 n-2
\end{array}\right)\right. \\
&+\sum_{l \equiv k(2), 0 \leqq l \leqq 2 k}\left(\begin{array}{c}
2 n-2+2 k-l \\
2 n-2
\end{array}\right)\left(\begin{array}{c}
2 n-2+l \\
2 n-2
\end{array}\right) \\
&\left.-\frac{1}{2} \sum_{0 \leqq l \leqq 2 k}\left(\begin{array}{c}
2 n-2+2 k-l \\
2 n-2
\end{array}\right)\left(\begin{array}{c}
2 n-2+l \\
2 n-2
\end{array}\right)\right\} \\
&= \frac{2 n-1+2 k}{2 n-1}\left\{\frac{1}{2} \sum_{l \equiv k(m), 0 \leqq l \leqq 2 k}\left(\begin{array}{c}
2 n-2+k+(k-l) \\
2 n-2
\end{array}\right)\left(\begin{array}{c}
2 n-2+k-(k-l) \\
2 n-2
\end{array}\right)\right. \\
&+\sum_{l \equiv k(2), 0 \leqq l \leqq 2 k}\left(\begin{array}{c}
2 n-2+k+(k-l) \\
2 n-2
\end{array}\right)\left(\begin{array}{c}
2 n-2+k-(k-l) \\
2 n-2
\end{array}\right) \\
&\left.-\frac{1}{2} \sum_{0 \leqq l \leqq 2 k}\left(\begin{array}{c}
2 n-2+k+(k-l) \\
2 n-2
\end{array}\right)\left(\begin{array}{c}
2 n-2+k-(k-l) \\
2 n-2
\end{array}\right)\right\} \\
&= \frac{2 n-1+2 k}{2 n-1}\left\{\begin{array}{c}
\sum_{t=1}^{[k / m]}\left(\begin{array}{c}
2 n-2+k+m t \\
2 n-2
\end{array}\right)\left(\begin{array}{c}
2 n-2+k-m t \\
2 n-2
\end{array}\right) \\
\end{array}\right. \\
&\left.+\sum_{l=0}^{k}(-1)^{l}\left(\begin{array}{c}
2 n-2+k+l \\
2 n-2
\end{array}\right)\left(\begin{array}{c}
2 n-2+k-l \\
2 n-2
\end{array}\right)\right\} .
\end{aligned}
$$

If $n=1$ and $k$ is odd, then the second term vanishes. Using Lemma 2.6, we have $a_{2 k}>0$ for $n>1$. Thus we have,

THEOREM 4.3. (1) The spectrum of Laplacian for the homogeneous spherical space form $S^{3} / \boldsymbol{D}_{4 m}^{*}(m>1)$ is

\begin{tabular}{|l|l|l|}
\hline$k$ & eigenvalue & multiplicity \\
\hline even & $4 k(k+1)$ & $(2 k+1)([k / m]+1)$ \\
\hline odd $k>m$ & $4 k(k+1)$ & $(2 k+1)[k / m]$ \\
\hline
\end{tabular}

(2) Eigenvalues of Laplacian for the homogeneous spherical space form $S^{4 n-1} / \boldsymbol{D}_{4 m}^{*}$ $(n, m>1)$ are 
with multiplictties

$$
4 k(2 n+k-1) \quad(k=0,1,2 \cdots)
$$

$$
\begin{gathered}
\frac{2 n-1+2 k}{2 n-1}\left\{\sum_{i=1}^{[k / m]}\left(\begin{array}{c}
2 n-2+k+m t \\
2 n-2
\end{array}\right)\left(\begin{array}{c}
2 n-2+k-m t \\
2 n-2
\end{array}\right)\right. \\
\left.+\sum_{l=0}^{k}(-1)^{l}\left(\begin{array}{c}
2 n-2+k+l \\
2 n-2
\end{array}\right)\left(\begin{array}{c}
2 n-2+k-l \\
2 n-2
\end{array}\right)\right\} .
\end{gathered}
$$

(III) $S^{4 n-1} / \boldsymbol{T}^{*}$.

By Proposition 3.1, $T^{*}$ consists of 4 cyclic subgroups of order 6 and 3 cyclic subgroups of order 4 . The intersection subgroup of any two of these cyclic subgroups is a cyclic group of order 2 . Thus we have

$$
\begin{aligned}
F_{T^{*}}(z) & =\frac{1}{24}\left\{24 F_{6}(2 n, z)+12 F_{4}(2 n, z)-12 F_{2}(2 n, z)\right\} \\
& =F_{6}(2 n, z)+\frac{1}{2} F_{4}(2 n, z)-\frac{1}{2} F_{2}(2 n, z)
\end{aligned}
$$

In this case, we have also $a_{2 k-1}=0(k \geqq 1)$ and

$$
\begin{aligned}
a_{2 k}= & \frac{2 n-1+2 k}{2 n-1}\left\{\sum_{l \equiv k(3), 0 \leq l \leq 2 k}\left(\begin{array}{c}
2 n-2+2 k-l \\
2 n-2
\end{array}\right)\left(\begin{array}{c}
2 n-2+l \\
2 n-2
\end{array}\right)\right. \\
& +\frac{1}{2} \sum_{l \equiv k(2), 0 \leqq l \leqq 2 k}\left(\begin{array}{c}
2 n-2+2 k-l \\
2 n-2
\end{array}\right)\left(\begin{array}{c}
2 n-2+l \\
2 n-2
\end{array}\right) \\
& \left.-\frac{1}{2} \sum_{0 \leq l \leq 2 k}\left(\begin{array}{c}
2 n-2+2 k-l \\
2 n-2
\end{array}\right)\left(\begin{array}{c}
2 n-2+l \\
2 n-2
\end{array}\right)\right\} \\
= & -\frac{2 n-1+2 k}{2 n-1}\left\{\sum_{l \equiv k(3), 0 \leq l \leq 2 k}\left(\begin{array}{c}
2 n-2+k+(k-l) \\
2 n-2
\end{array}\right)\left(\begin{array}{c}
2 n-2+k-(k-l) \\
2 n-2
\end{array}\right)\right. \\
& +\frac{1}{2} \sum_{l \equiv k(2), 0 \leqq l \leqq 2 k}\left(\begin{array}{c}
2 n-2+k+(k-l) \\
2 n-2
\end{array}\right)\left(\begin{array}{c}
2 n-2+k-(k-l) \\
2 n-2
\end{array}\right) \\
& \left.-\frac{1}{2} \sum_{0 \leqq l \leqq 2 k}\left(\begin{array}{c}
2 n-2+k+(k-l) \\
2 n-2
\end{array}\right)\left(\begin{array}{c}
2 n-2+k-(k-l) \\
2 n-2
\end{array}\right)\right\} \\
= & \frac{2 n-1+2 k}{2 n-1}\left\{\left(\begin{array}{c}
2 n-2+k \\
2 n-2
\end{array}\right)^{2}+2 \sum_{t=1}^{[k / 3]}\left(\begin{array}{c}
2 n-2+k+3 t \\
2 n-2
\end{array}\right)\left(\begin{array}{c}
2 n-2+k-3 t \\
2 n-2
\end{array}\right)\right. \\
& +\sum_{t=1}^{[k / 2]}\left(\begin{array}{c}
2 n-2+k+2 t \\
2 n-2
\end{array}\right)\left(\begin{array}{c}
2 n-2+k-2 t \\
2 n-2
\end{array}\right) \\
& \left.-\sum_{t=1}^{k}\left(\begin{array}{c}
2 n-2+k+t \\
2 n-2
\end{array}\right)\left(\begin{array}{c}
2 n-2+k-t \\
2 n-2
\end{array}\right)\right\} .
\end{aligned}
$$

If $n>1$ then by Lemma 2.6, we see easily that $a_{2 k}>0$. If $n=1$, then $a_{2 k}=$ $(2 k+1)(1+2[k / 3]+[k / 2]-k)$. Thus we have 
THEOREM 4.4. (1) The spectrum of Laplacian for the homogeneous spherical space form $S^{3} / T^{*}$ is

\begin{tabular}{|l|c|c|}
\hline$k \geqq 0$ & eigenvalue & multıplicity \\
\hline$k \neq 1,2,5$ & $4 k(k+1)$ & $(2 k+1)(1+2[k / 3]+[k / 2]-k)$ \\
\hline
\end{tabular}

(2) Eigenvalues of Laplacian for the homogeneous spherical space form $S^{4 n-1} / \boldsymbol{T}^{*}$ $(n>1)$ are

$$
4 k(2 n+k-1) \quad(k=0,1,2 \cdots)
$$

with multiplicities

$$
\begin{aligned}
& \frac{2 n-1+2 k}{2 n-1}\left\{\left(\begin{array}{c}
2 n-2+k \\
2 n-2
\end{array}\right)^{2}+2 \sum_{t=1}^{[k / 3]}\left(\begin{array}{c}
2 n-2+k+3 t \\
2 n-2
\end{array}\right)\left(\begin{array}{c}
2 n-2+k-3 t \\
2 n-2
\end{array}\right)\right. \\
& \quad+2 \sum_{t=1}^{[k / 2]}\left(\begin{array}{c}
2 n-2+k+2 t \\
2 n-2
\end{array}\right)\left(\begin{array}{c}
2 n-2+k-2 t \\
2 n-2
\end{array}\right) \\
& \left.\quad-\sum_{t=1}^{k}\left(\begin{array}{c}
2 n-2+k+t \\
2 n-2
\end{array}\right)\left(\begin{array}{c}
2 n-2+k-t \\
2 n-2
\end{array}\right)\right\} .
\end{aligned}
$$

(IV) $S^{4 n-1} / O^{*}$.

By Proposition 3.1, $\boldsymbol{O}^{*}$ consists of 3 cyclic subgroups of order 8,4 cyclic subgroups of order 6 and cyclic groups of order 4 . The intersection subgroup of any two of these cyclic subgroups is a cyclic group of order 2. Thus we have

$$
\begin{aligned}
F_{O *}(z) & =\frac{1}{48}\left\{24 F_{8}(2 n, z)+24 F_{6}(2 n, z)+24 F_{4}(2 n, z)-24 F_{2}(2 n, z)\right\} \\
& =\frac{1}{2}\left\{F_{8}(2 n, z)+F_{6}(2 n, z)+F_{4}(2 n, z)-F_{2}(2 n, z)\right\} .
\end{aligned}
$$

By the same calculations as in (III), we have

THEOREM 4.5. (1) The spectrum of Laplacian for the homogeneous spherical space form $S^{3} / O^{*}$ is

\begin{tabular}{|l|l|c|}
\hline$k \geqq 0$ & eigenvalue & multiplicity \\
\hline$k \neq 1,2,3,5,7,11$ & $4 k(k+1)$ & $(2 k+1)(1+[k / 4]+[k / 3]+[k / 2]-k)$ \\
\hline
\end{tabular}

(2) Eigenvalues of Laplacian for the homogeneous spherical space form $S^{4 n-1} / \mathrm{O}^{*}$ $(n>1)$ are

$$
4 k(2 n+k-1) \quad(k=0,1,2 \cdots)
$$

with multiplicitıes 


$$
\begin{aligned}
& \frac{2 n-1+2 k}{2 n-1}\left\{\left(\begin{array}{c}
2 n-2+k \\
2 n-2
\end{array}\right)^{2}+\sum_{t=1}^{[k / 4]}\left(\begin{array}{c}
2 n-2+k+4 t \\
2 n-2
\end{array}\right)\left(\begin{array}{c}
2 n-2+k-4 t \\
2 n-2
\end{array}\right)\right. \\
& +\sum_{t=1}^{[k / 3]}\left(\begin{array}{c}
2 n-2+k+3 t \\
2 n-2
\end{array}\right)\left(\begin{array}{c}
2 n-2+k-3 t \\
2 n-2
\end{array}\right) \\
& +\sum_{t=1}^{[k / 2]}\left(\begin{array}{c}
2 n-2+k+2 t \\
2 n-2
\end{array}\right)\left(\begin{array}{c}
2 n-2+k-2 t \\
2 n-2
\end{array}\right) \\
& \left.\quad-\sum_{t=1}^{k}\left(\begin{array}{c}
2 n-2+k+t \\
2 n-2
\end{array}\right)\left(\begin{array}{c}
2 n-2+k-t \\
2 n-2
\end{array}\right)\right\} .
\end{aligned}
$$

(V) $S^{4 n-1} / I^{*}$. By Proposition 3.1, I* consists of 6 cyclic subgroups of order 10, 10 cyclic subgroups of order 6 and 15 cyclic groups of order 4 . The intersection sugroup of any two of these cyclic subgroups is a cyclic group of order 2. Thus we have

$$
\begin{aligned}
F_{I *}(z) & =\frac{1}{120}\left\{60 F_{10}(2 n, z)+60 F_{6}(2 n, z)+60 F_{4}(2 n, z)-60 F_{2}(2 n, z)\right\} \\
& =\frac{1}{2}\left\{F_{10}(2 n, z)+F_{6}(2 n, z)+F_{4}(2 n, z)-F_{2}(2 n, z)\right\} .
\end{aligned}
$$

By the same calculations as in (III), we have

THEOREM 4.6. (1) The spectrum of Laplacian for the homogeneous spherical space form $S^{3} / I^{*}$ is

\begin{tabular}{|l|c|}
\hline eigenvalue & multıplicity \\
\hline $4 k(k+1)$ & $(2 k+1)(1+[k / 5]+[k / 3]+[k / 2]-k)$ \\
\hline
\end{tabular}

where $k$ runs through all nonnegative integers except $1,2,3,4,5,7,8,9,11,13$, 14, 17, 19, 23, 29. (2) Eigenvalues of Laplacian for the homogeneous spherical space form $S^{4 n-1} / I^{*}(n>1)$ are

with multiplicities

$$
4 k(2 n+k-1) \quad(k=0,1,2 \cdots)
$$

$$
\begin{aligned}
& \frac{2 n-1+2 k}{2 n-1}\left\{\left(\begin{array}{c}
2 n-2+k \\
2 n-2
\end{array}\right)^{2}+\sum_{t=1}^{[k / 5]}\left(\begin{array}{c}
2 n-2+k+5 t \\
2 n-2
\end{array}\right)\left(\begin{array}{c}
2 n-2+k-5 t \\
2 n-2
\end{array}\right)\right. \\
& +\sum_{t=1}^{[k / 3]}\left(\begin{array}{c}
2 n-2+k+3 t \\
2 n-2
\end{array}\right)\left(\begin{array}{c}
2 n-2+k-3 t \\
2 n-2
\end{array}\right) \\
& +\sum_{t=1}^{[k / 2]}\left(\begin{array}{c}
2 n-2+k+2 t \\
2 n-2
\end{array}\right)\left(\begin{array}{c}
2 n-2+k-2 t \\
2 n-2
\end{array}\right) \\
& \left.-\sum_{t=1}^{k}\left(\begin{array}{c}
2 n-2+k+t \\
2 n-2
\end{array}\right)\left(\begin{array}{c}
2 n-2+k-t \\
2 n-2
\end{array}\right)\right\} .
\end{aligned}
$$




\section{First non zero eigenvalues}

Using the results of 4 , we can obtain the first non zero eigenvalues of homogeneous spherical space forms.

THEOREM 5.1. The first non zero eigenvalue of Laplacian for homogeneous spherical space forms are

(1) 3-dimensional spherical space forms.

\begin{tabular}{|l|c|c|}
\hline & 1st non zero eigenvalue & multiplicity \\
\hline$S^{3} / \boldsymbol{Z}_{2}$ & 8 & 9 \\
\hline$S^{3} / \boldsymbol{Z}_{q} \quad q>2$ & 8 & 3 \\
\hline$S^{3} / \boldsymbol{D}_{4 m}^{*} \quad m \geqq 2$ & 24 & 3 \\
\hline$S^{3} / \boldsymbol{T}^{*}$ & 48 & 7 \\
\hline$S^{3} / \boldsymbol{O}^{*}$ & 80 & 9 \\
\hline$S^{3} / \boldsymbol{I}^{*}$ & 168 & 13 \\
\hline
\end{tabular}

(2) Spherical space forms $S^{2 n-1} / G(n>2)$.

\begin{tabular}{|l|c|c|}
\hline & 1 st eigenvalue & multiplicity \\
\hline$S^{2 n-1} / \boldsymbol{Z}_{2}$ & $4 n$ & $(n+1)(2 n-1)$ \\
\hline$S^{2 n-1} / \boldsymbol{Z}_{q} \quad(q>2)$ & $4 n$ & $(n-1)^{2}$ \\
\hline$S^{4 n-1} / \boldsymbol{D}_{4 m}^{*} \quad(m \geqq 2)$ & $8 n$ & $(n-1)(2 n+1)$ \\
\hline$S^{4 n-1} / \boldsymbol{T}^{*}$ & $8 n$ & $(n-1)(2 n+1)$ \\
\hline$S^{4 n-1} / \boldsymbol{O}^{*}$ & $8 n$ & $(n-1)(2 n+1)$ \\
\hline$S^{4 n-1} / \boldsymbol{I}^{*}$ & $8 n$ & $(n-1)(2 n+1)$ \\
\hline
\end{tabular}

\section{REFERENCES}

[1] B. Berger, Le Spectre d'une Variétés Riemannienne, Lecture Notes in Math., 194 Springer-Verlag, 1971.

[2] A. IKEDA, On spherical space forms which are isospectral but not isometric, J. Math. Soc. Japan, 35 (1983), 437-444. 
[3] T. SAKaI, On the spectrum of lens spaces, Kōdai Math. Sem. Rep., 27 (1976), 249-257.

[4] J.A. Wolf, Spaces of Constant Curvature, McGraw-Hill, 1967.

Department of Mathematics

FACUlTy OF School Education

HiRoshima UNIVERSITY

SHINONOME HiRoshima, 734

Japan 